\title{
Intensity Modulated Radiotherapy Using Multiple in Fields Compared to Wedged Fields in Breast Irradiation: Clinical Impact on Early Skin Reaction and Organs at Risk Doses
}

\author{
Hala A Zaghloul ${ }^{1,2^{\star}}$, Yasser A Rashed ${ }^{1,3}$, Arif A Shaukat ${ }^{1}$, Yousry A Rostom ${ }^{2}$ and Samy E Badawy ${ }^{1}$ \\ ${ }^{1}$ Radiation Oncology, King Fahad Specialist Hospital Al Dammam, KSA \\ ${ }^{2}$ Clinical Oncology, Alexanderia University, Egypt \\ ${ }^{3}$ Radiation Oncology and nuclear medicine Dept., Faculty of medicine, Menofiya University, Egypt
}

*Corresponding author: Hala Ahmed Zaghloul El Lathy, Department of Radiation Oncology, king Fahad Specialist Hospital Al Dammam KSA \& Clinical Oncology, Alexanderia University, Egypt, Tel: +00966 540 367076; Fax: 009668442222 Ext : 5851; E-mail: h_zagloul@yahoo.com

Received date: Apr 10, 2014, Accepted date: June 20, 2014, Publication date: June 30, 2014

Copyright: ( 2014 Zaghloul HA, et al. This is an open-access article distributed under the terms of the Creative Commons Attribution License, which permits unrestricted use, distribution, and reproduction in any medium, provided the original author and source are credited.

\author{
Abstract \\ Objective: To measure the impact of multiple fields in filed (MFIF) technique on dose homogeneity, doses to \\ organs at risk (OARs) and acute skin toxicity in comparison to physical wedged (PW) tangential technique.
}

Methods: The study population consisted of 75 patients who had breast-conserving surgery and whole-breast radiation using MFIF. These patients were matched one-to-one to a control group of 75 patients treated with $\mathrm{PW}$ tangents, following ethical board approval and signing informed consents. Acute skin reaction was graded by CTCAE 3.0 scoring and multiple regression analysis of covariates was performed.

Results: CTCAE grade2 acute skin reaction occurred in $61.3 \%$ PW vs. $32 \%$ MFIF while grade 3 was encountered in $24 \%$ PW vs. $10.7 \%$ MFIF patients $(P<0.01)$. Multiple regression analysis confirmed the excessive skin reaction to the related radiation therapy technique $(P=0.0002)$ and whole breast $P T V(P<0.001)$. Homogeneity index within PTV was significantly improved in MFIF plans $(0.84 \pm 0.7)$ compared with $\mathrm{PW}(0.94 \pm 0.15)$ with a $(\mathrm{P}<0.01)$. There was also a significant reduction, in lung V20 from $14.3 \% \pm 1.1$ to $10.6 \% \pm 1.4(P<0.01)$, heart V30 from $7 \% \pm 5.8$ to $6 \% \pm 4.9(p<0.001)$. Contralateral breast volume receiving dose more than 2 Gy was also reduced $2.3 \% \pm 0.5 \mathrm{PW}$ to $1.2 \% \pm 0.2 \mathrm{MFIF}(\mathrm{P}<0.01)$.

Conclusion: The MFIF technique significantly improved PTV dose homogeneity index, while significantly reduced the incidence of grade $2 / 3$ acute skin reaction and doses to OARs when compared to PW tangential fields in whole breast radiotherapy.

Keywords: Intensity modulated radiation; Multiple in fields whole breast irradiation

\section{Introduction}

The three-dimensional analysis of tangential parallel-opposed pair technique used for whole breast irradiation (WBI) demonstrated large dose inhomogeneity inside the target volume resulting in hot spots with increased risk of adverse effects and inferior cosmetic outcome [1-3]. Moreover, achieving acceptable dose homogeneity across the whole breast volume is difficult because of the continuous change in breast shape across multiple planes [4]. Various techniques have been tested in an attempt to improve the dose distribution in the PTV as physical wedged tangential (PW) systems and intensity modulation techniques [5-10].

The use of 3D treatment planning and intensity modulation with static multileaf collimator (sMLC) technique for tangential whole breast RT was found to be an efficient and reliable method for achieving a uniform dose throughout the whole breast. Strict dosevolume constraints can be readily achieved in most patients, resulting in both improved coverage of breast tissue, as well as a potential reduction in acute and chronic toxicities [4-6]. Moreover, several studies have reported improvement in the dose homogeneity with better sparing of OARs, when inverse planning intensity modulation radiation (IMRT) techniques were applied [11-15]. Additionally, moist desquamations were reported in $31 \%$ of patients treated with inverse IMRT versus $48 \%$ of patients treated with conventional wedged tangents [13]. However, the workload for planning and delivering inverse IMRT is significant compared to conventional PW, with an increase in rad-on time leading to higher bone marrow exposure to scattered doses $[13,14]$. The rationale of using MFIF was based on its improved performance compared with the conventional physical wedge (PW) and dynamic wedge systems [15]. Additionally, MFIF using beam 's eye view (BEV) isodose-contouring and equalization of the maximum dose achieved the best reduction of high-dose volumes, without clear dosimetric advantage of other methods of intensity modulation including inverse IMRT over it [10].

The purpose of the study is to measure the impact of multiple fields in filed (MFIF) technique on dose homogeneity, doses to organs at risk (OARs) and acute skin toxicity in comparison to physical wedged (PW) tangential technique. 
Citation: Zaghloul HA, Rashed YA, Shaukat AA, Rostom YA, Badawy SE (2014) Intensity Modulated Radiotherapy Using Multiple in Fields Compared to Wedged Fields in Breast Irradiation: Clinical Impact on Early Skin Reaction and Organs at Risk Doses. J Nucl Med Radiat Ther 5: 176. doi:10.4172/2155-9619.1000176

Page 2 of 8

\section{Methods}

In this retrospective study which was approved by the ethical committee in our institute, the study population consisted of 75 consecutive patients who had breast-conserving surgery and wholebreast radiation using MFIF between May 2009 - December 2011. These patients were individually matched (one-to-one) to a control group of 75 patients treated with PW tangents during the same period. The PW (control) subjects were selected to broadly match the distribution of age groups, clinical stage, whole breast PTV volumes, hormones and chemotherapy of the MFIF (study) group. Binary logisitic regression was run using radiation field technique the PW (control) and the MFIF (study) as a binary dependent variable to calculate the probability predictive value which was used to match (control and study groups).

All patients signed informed consent before radiation. We excluded patients treated with implanted prosthesis, postoperative wound infection, and previous radiation to the same breast, connective tissue disorder or diabetes from both groups.

We opted to evaluate effectiveness of MFIF technique over PW in whole breast irradiation by comparing treatment dosmetric parameters and acute skin reactions.

We also opted to assess the impact of both (MFIF \& PW) techniques on skin dose. Surface doses were measured in ten patients in each treatment group (MFIF \& PW) using Lithium fluoride TLD chips (TLD-100 (Saint-Gobain Crystals \& Detectors, Newbury, OH) with dimensions of $3.0 \times 3.0 \times 0.9 \mathrm{~mm}^{3}$. Four evenly spaced TLD chips were placed on the surface of each breast along the positive and the negative $\mathrm{x}$-axis relative to the central axis of the beam. Measurements on both breasts were taken at equal intervals starting from $3 \mathrm{~cm}$ from the geometric edge, closest to the penumbra region of the beam. Their evaluation was carried out in a PCL3 TL reader of the FIMEL Company (Fontenay Aux Roses, France). TLDs were consistently annealed at $400^{\circ} \mathrm{C}$ for $1 \mathrm{~h}$ and $100^{\circ} \mathrm{C}$ for $2 \mathrm{~h}$ prior to irradiation and $100^{\circ} \mathrm{C}$ for $10 \mathrm{~min}$ after irradiation for pre-readout annealing.

\section{Treatment Planning}

All patients were scanned using Computed Tomography (CT). The breast CTV with $0.7 \mathrm{~cm}$ margin to PTV were outlined on each CT simulation image of $5 \mathrm{~mm}$ thickness each using Eclipse version 8.6 (TPS). Additionally, regional lymph nodes and organs at risk including both lungs, heart and spinal cord were also contoured for treatment planning and dose volume histogram (DVHs) generation. Finally the CTV boost was created to include the tumour bed and any seroma and a $1.5-2 \mathrm{~cm}$ margin in all directions to create PTV boost, editing 2-3 $\mathrm{mm}$ from the skin and lung surfaces.

A reference point was created as recommended by ICRU 50 convention as a point on central axis midway on a perpendicular line between the breast apex and the posterior field edge on designing medial and lateral tangential fields [16].

For generating MFIF plans, beam's eye view (BEV) isodosecontouring and equalization of the maximum dose was applied. A dose distribution without any beam modifier was first created. The dose distribution based on open beam fields had been evaluated to lower maximum doses $>107 \%$ within the PTV and to eliminate the hot spot volumes. Maximum target dose is defined as the highest dose in the target area provided this dose covers a minimum area of $2 \mathrm{~cm}^{2}$. While hotspots are defined as an area outside the target that receives a higher dose than the specified target dose. It is considered clinically meaningful only if it covers an area of at least $2 \mathrm{~cm}^{2}$ [17]. Using the isodose display features of the planning system for the $>107 \%$ isodoses, multileaf collimator (MLC) segments were used to design blocking subfields (segments) to improve the dose homogeneity within the PTV. Main fields and subfields were merged in one portal, including several MLC segments for sequential irradiation. For patients treated using PW medial and lateral wedged tangential beams were used and the applied angles ranged from 15 to 30 degrees. Photon beam of 6 and $18 \mathrm{MV}$ photon beam (Varian $2300 \mathrm{CD}$ multimodality with 120-leaf Millennium) were used depending on patient separation.

A correction for lung inhomogeneity was made for all patients. The maximum, mean, and minimum dose values of PTV of MFIF and PW treated patients were compared using their DVHs. We also analysed the DVHs of ipsilateral lung, heart and contralateral breast for each patient. Moreover, we compared the total average monitor units (MUs) of MFIF to PW treated patients. The homogeneity index (HI) for each plan was calculated using the following formula and the mean values were compared.

Homogeneity index $(\mathrm{HI})=($ Dose Max - Dose Min $) /$ Dose Mean in PTV

The significance of $\mathrm{HI}$ is that a lesser value of $\mathrm{HI}$ indicates greater 3D dose homogeneity in the planned target volume (PTV) [18].

Volumes over $20 \mathrm{~Gy}$ in the ipsilateral lung, $40 \mathrm{~Gy}$ in the heart and 2 Gy in the contralateral breast were used to establish the extent of the high dose received by the OARs.

\section{Treatment verification and quality assurance}

Quality assurance was performed using $2 \mathrm{D}$ array system. It is COMPASS software with the IMRT Matrix which is the most advanced solution for fast, accurate and reliable digital IMRT verification.

\section{Radiotherapy Dose Prescription}

Whole breast \pm nodal areas radiotherapy was delivered as $50 \mathrm{~Gy}$ in 25 fractions over 5 weeks. A breast boost of $10 \mathrm{~Gy}$ in 5 daily fractions was delivered to all patients using a direct electron beam. The depth of the target boost volume, should be encompassed by the 90 per cent isodose. Electrons of 9-15 MeV may be required but exit doses to the heart should be avoided. For larger volumes, small tangential photon beams $6 \mathrm{MV}$ were devised.

\section{Clinical Assessments of the Breast}

Archived original datasets of each patient actually applied treatment plan (MFIF and PW) were reviewed by the radiation oncologist and the medical physicist conducting the study. The grade of acute skin reaction and its location in the breast were retrieved from recorded weekly patient assessment during the 5 to 6 weeks of RT and at weeks $1,2,4$, and 6 after treatment by treating physician. Acute skin reactions were scored according to the radiation oncologists' records applying the CTCAE 3.0 scoring system [19]. We also observed the isodose distribution for MFIF and PW treated patients and recorded the location of high dose regions (maximum target doses and hot spots) as shown on the isodose distribution. The location for moist desquamation was also recorded to see if it matches the high dose regions. 
Citation: Zaghloul HA, Rashed YA, Shaukat AA, Rostom YA, Badawy SE (2014) Intensity Modulated Radiotherapy Using Multiple in Fields Compared to Wedged Fields in Breast Irradiation: Clinical Impact on Early Skin Reaction and Organs at Risk Doses. J Nucl Med Radiat Ther 5: 176. doi:10.4172/2155-9619.1000176

Page 3 of 8

\section{Statistical analysis}

All statistical analysis was performed using the SPSS software (version 13).

Baseline data were compared using the student t-test, Pearson $\chi^{2}$ test for heterogeneity and trend the Fisher Exact test was used. The dosimetry data were summarized using means and standard deviations and comparisons were made using the independent sample $t$-test. Moreover, 95\% confidence intervals (CI were quoted with all P-values. Linear regression was performed to assess the relationship between acute skin reaction and radiation therapy technique (PW and MFIF, maximum dose with in the PTV and whole breast PTV volume) Sample size was calculated using published tables for $\pm 5 \%$ precision Levels, where $(\mathrm{CI})$ is $95 \%$ and maximum degree of variability is $\mathrm{P}=0$. 05 .

\begin{tabular}{|c|c|c|c|}
\hline & Multiple field in field (Study group) & Physical Wedge (Control group) & $P$ Value \\
\hline & $N=75$ & $\mathrm{~N}=\mathbf{7 5}$ & \\
\hline \multicolumn{4}{|l|}{ Age groups } \\
\hline$>25-<35$ & $5(6.7 \%)$ & $5(6.7 \%)$ & 0.065 \\
\hline$>35-<45$ & $24(32 \%)$ & $29(38.7 \%)$ & \\
\hline$>45-<55$ & $36(48 \%)$ & $23(30.7 \%)$ & \\
\hline$>55-<65$ & $5(6.7 \%)$ & $15(20 \%)$ & \\
\hline$>65-<75$ & $5(6.7 \%)$ & $3(4 \%)$ & \\
\hline \multicolumn{4}{|l|}{ TNM Stage } \\
\hline Stage I & $43(57.3 \%)$ & $32(42.7 \%)$ & \\
\hline Stage IIA & $28(37.3 \%)$ & $38(50.7 \%)$ & \\
\hline Stage IIB & $2(2.7 \%)$ & $3(4 \%)$ & 0.344 \\
\hline Stage IIIA & $2(2.7 \%)$ & $2(2.7 \%)$ & \\
\hline \multicolumn{4}{|l|}{ Chemotherapy } \\
\hline No Chemotherapy & \multirow{2}{*}{$\begin{array}{l}4(5.3 \%) \\
40(53.3 \%)\end{array}$} & $0(0 \%)$ & \\
\hline AC X 4 Cycle & & $50(66.7 \%)$ & 0.211 \\
\hline $\begin{array}{l}\text { ACX4 Cycles }+ \\
\text { Taxotere } x 4 \text { Cycles }\end{array}$ & $22(29.3 \%)$ & $16(21.3 \%)$ & \\
\hline FEC X6Cycles & $2(2.7 \%)$ & $2(2.7 \%)$ & \\
\hline TC $\times 6$ cycles & $0(0 \%)$ & $1(1.3 \%)$ & \\
\hline $\begin{array}{l}\text { ACX4 Cycles + Herceptin } \\
\text { Taxotere } x 4 \text { Cycles }\end{array}$ & $7(9.3 \%)$ & $6(8 \%)$ & \\
\hline Hormones & & & 0.152 \\
\hline No Hormones & $20(26.3 \%)$ & $14(18.7 \%)$ & \\
\hline Tamoxifen & $47(62.7 \%)$ & $45(60 \%)$ & \\
\hline Letrozole & $8(10.7 \%)$ & $16(21.3 \%)$ & \\
\hline $\begin{array}{l}\text { Mean Whole Breast } \\
\text { PTV Volume } \pm \text { SD }\end{array}$ & $1778.2 \mathrm{cc} \pm 838$ & $1705.4 \mathrm{cc} \pm 889$ & 0.103 \\
\hline
\end{tabular}

Table 1: Patient and treatment characteristics of multiple fields in field (MFIF) study group and the physical wedge (PW) control group.

\section{Results}

On comparing demographic characteristics and treatment parameters of MFIF treated patients to those treated with PW, no statistical significant was observed as summarized in Table 1 . The median age was 48 years (range 28-68). The mean volume for the whole breast PTV was $1778.2 \mathrm{cc}$ (range 380-3560 cc). There was no statistically significant difference between breast volumes in the two groups $(\mathrm{P}=0.103)$. In MFIF 1 to 4 static fields were planned within the two main tangential fields to achieve adequate dose distribution. Beam weights assigned to these segmented fields were between $5 \%$ and $7 \%$ of the total isocentric weight of the open beams. While in the PW, the wedge angles used were either $15^{\circ}$ or $30^{\circ}$.There was a significant reduction in the total MUs delivered to medial and lateral tangential fields in the MFIF $122.3 \pm 16$ and $121.6 \pm 18$ MUs when compared .

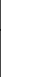


Citation: Zaghloul HA, Rashed YA, Shaukat AA, Rostom YA, Badawy SE (2014) Intensity Modulated Radiotherapy Using Multiple in Fields Compared to Wedged Fields in Breast Irradiation: Clinical Impact on Early Skin Reaction and Organs at Risk Doses. J Nucl Med Radiat Ther 5: 176. doi:10.4172/2155-9619.1000176

Page 4 of 8

Comparison of dosimetric parameters as (breast separation, beam energies used in both tangent and boost fields) did not illustrate significant difference between the MFIF and the PW groups as summarized in Table 2. Moreover, there was no statistically significant difference in the dose delivered to $95 \%$ of the breast PTV between MFIF compared to PW ( $98 \%$ vs $99 \%$ ) of prescribed dose respectively $(P$ value $=0.6)$.

\begin{tabular}{|l|l|l|l|}
\hline & $\begin{array}{l}\text { Multiple Fields in } \\
\text { Fields }\end{array}$ & Physical wedge & P Value \\
\cline { 2 - 4 } & (study group) & (control group) & \\
\cline { 2 - 4 } & $\mathbf{N = 7 5}$ & $\mathbf{N = 7 5}$ & \\
\hline $\begin{array}{l}\text { Breast separation in } \\
\text { the central axis }\end{array}$ & & & \\
\hline$\geq 23 \mathrm{Cm}$ & $15(20 \%)$ & $18(24 \%)$ & \\
\hline$\geq 21-<23 \mathrm{Cm}$ & $16(21.3 \%)$ & $11(14.7 \%)$ & 0.099 \\
\hline$<21$ & $44(58.7 \%)$ & $16(61.3 \%)$ & \\
\hline Tangent Beam Energy & & & \\
\hline $\begin{array}{l}\text { Mixed 6 \& 18 MV } \\
\text { photons }\end{array}$ & $21(28 \%)$ & $24(32 \%)$ & \\
\hline $18 \mathrm{MV}$ & $46(61.3 \%)$ & $45(60 \%)$ & 0.45 \\
\hline 6 MV & $8(10.7 \%)$ & $6(8 \%)$ & \\
\hline Boost Energy & & & \\
\hline Electron (9-15 MEV) & $60(80 \%)$ & $58(77.3 \%)$ & 0.150 \\
\hline Mini-Tangent photon & $15(20 \%)$ & $17(22.7 \%)$ & \\
\hline
\end{tabular}

Table 2: Comparison of dosimetric parameters between MFIF and PW patients.

The maximum target dose covering the PTV was significantly reduced in MFIF plans $107 \pm 1.5$ compared to $109 \pm 2.5$ in PW plans with a $(95 \% \mathrm{CI}$ for difference of $1.1-2.5)$ and a $(\mathrm{P}<0.01)$ ( Figure 1 and 2). Moreover, there was also a significant reduction in the hot spot values in the MFIF when compared to PW field plans $108 \% \pm 1.5$ vs $110 \% \pm 2.9)$ with a $(\mathrm{P}=0.008)$. Additionally, the mean PTV dose was reduced from $101.1 \% \pm 0.9$ in $\mathrm{PW}$ plans to $100.3 \% \pm 0.8$ in MFIF $(\mathrm{P}<0.0001)$ showing better dose distribution coverage in the PTV with MFIF plan. Consequently, significantly greater 3D dose homogeneity in the PTV was confirmed by the lesser value of HI for MFIF plans $0.84 \pm 0.7$ compared to PW plans $0.94 \pm 0.15$ with $(95 \%$ CI of difference of $0.92-0.125)$ and $(\mathrm{P}<0.01)$.

With regards to the impact of MFIF and PW techniques on skin surface dose, measured by TLD, it was observed that skin doses with both techniques were less than the daily prescribed therapeutic dose of 200cGy . MFIF delivered slightly higher mean doses on the surface of the treated breasts than PW (129.5 cGy vs 123.4 cGy respectively). On the other hand, mean surface doses to the contralateral breast were higher with PW (13.7 cGy) compared to MFIF (11.4cGy) treated patients as shown in Table 3 .

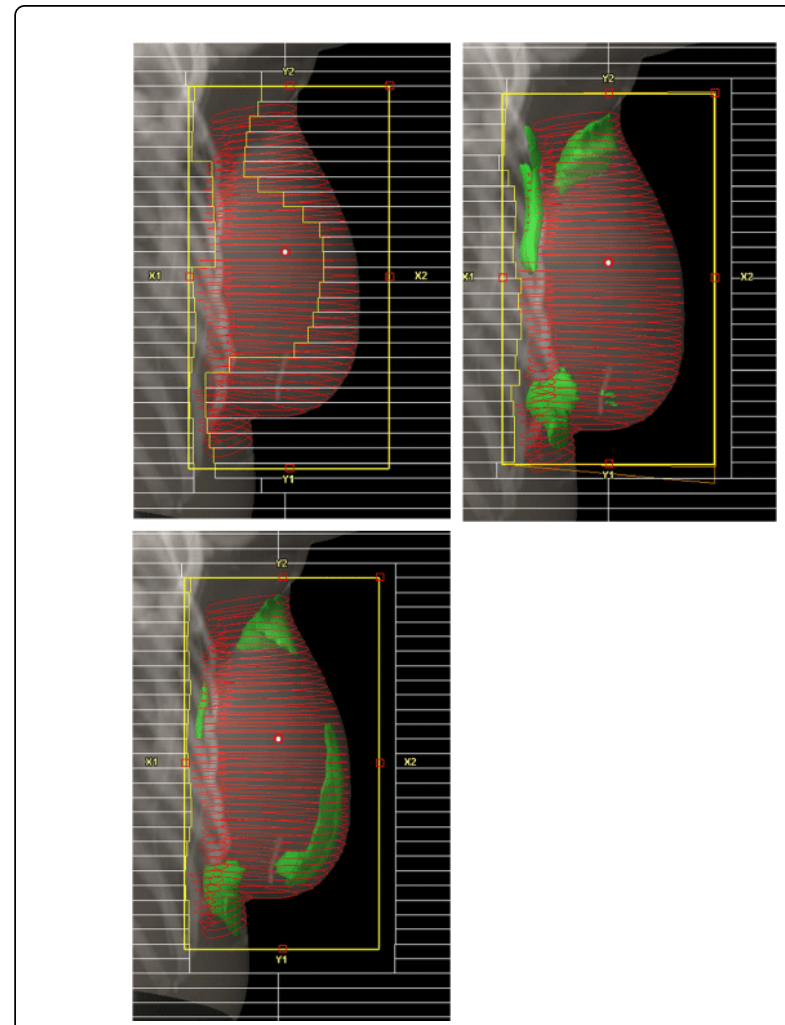

Figure 1: Beam`s eye view images. A: Open tangential field (left superior) showing Maximum target dose in green occupying large volume of breast PTV in red . B: Physical wedge (right superior) resulted in reduction in the Maximum target dose volume in breast PTV . C: Field in field (left inferior) resulted in almost complete disappearance of Maximum target dose from breast PTV.

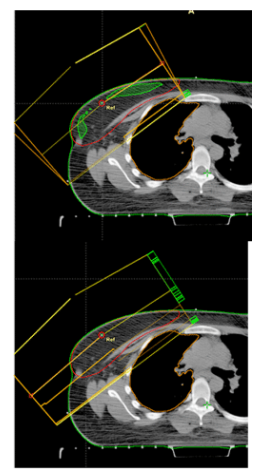

Figure 2: Showing axial CT simulation images. A: Physical wedge (superior) showing Maximum target dose in green occupying large volume of breast PTV in red. B: Field in field (inferior) resulted in complete disappearance of Maximum target dose from breast PTV.

$10.6 \% \pm 1.4$ vs $14.3 \% \pm 1.1(\mathrm{P}<0.01)$ and $6 \% \pm 4.9$ vs $7 \% \pm 5.8$ $(\mathrm{P}<0.001)$ respectively. Moreover, The PTV volume covered by more than $107 \%$ of the dose was reduced $(5.2 \% \pm 1$ vs $8.4 \% \pm 1.2$ respectively, $\mathrm{P}<0.01)$ for MFIF compared to $\mathrm{PW}$ plans. More interestingly, the percent volume of the contralateral breast receiving 
Citation: Zaghloul HA, Rashed YA, Shaukat AA, Rostom YA, Badawy SE (2014) Intensity Modulated Radiotherapy Using Multiple in Fields Compared to Wedged Fields in Breast Irradiation: Clinical Impact on Early Skin Reaction and Organs at Risk Doses. J Nucl Med Radiat Ther 5: 176. doi:10.4172/2155-9619.1000176

Page 5 of 8

over 2 Gy was significantly reduced $(1.2 \pm 0.2$ vs to $2.3 \pm 0.5)$ in MFIF compared to $\mathrm{PW}$ plans respectively $(\mathrm{P}<0.01)$ (Table 4$)$.

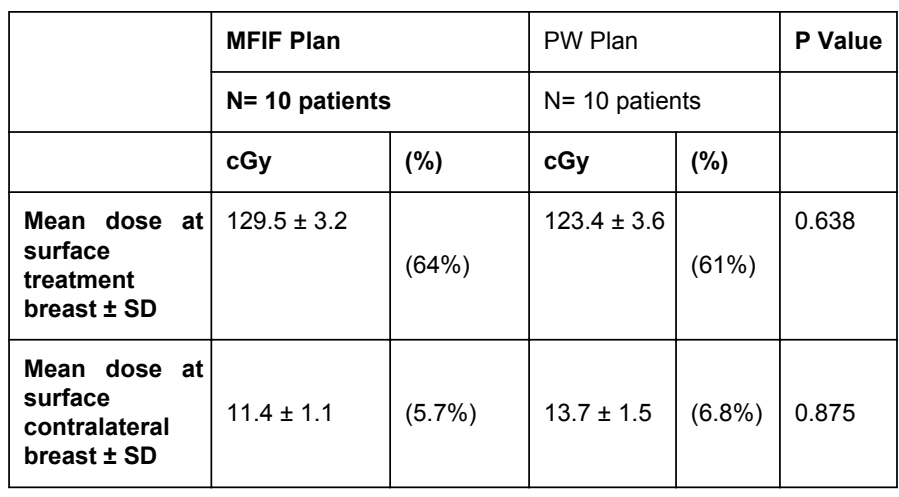

Table 3: Results of TLD readings; Therapeutic dose delivered for each plan was 200cGy. Numbers in parentheses are \% of total dose delivered.

Taking into consideration organs at risk: lung V20, heart V30 in the MFIF group of patients were significantly reduced compared to the PW group of patients.

Using the IMRT verification system 2D Array, the Gamma Index was in the range from $99.78 \%$ to $99.98 \%$ and the average is $99.89 \%$. More interestingly, the application of MFIF technique resulted in significant reduction in the incidence of acute skin reaction compared to PW. Grade 2 and 3 (moist desquamation) acute skin reaction were detected in 46 patients $(61.3 \%)$ and 18 patients $(24 \%)$ of patients treated using PW compared to 24 patients (32\%) and only 8 patients $(10.7 \%)$ of MFIF respectively $(\mathrm{P}<0.01)$. Additionally, grade 3 skin reaction (moist desquamation) was encountered in 16 patients $(88.9 \%)$ of the group with whole breast PTV volume $>2700 \mathrm{cc}<3700$ treated

\begin{tabular}{|c|c|c|c|c|c|c|c|c|}
\hline \multirow[t]{3}{*}{ Locations of high dose regions } & \multicolumn{4}{|c|}{ Distribution of high dose regions } & \multicolumn{4}{|c|}{$\begin{array}{l}\text { Moist desquamation occurred at the same area of the high } \\
\text { dose region }\end{array}$} \\
\hline & \multicolumn{2}{|c|}{ Inside breast } & \multicolumn{2}{|c|}{ Outside breast } & \multicolumn{2}{|l|}{ MFIF (8) } & \multicolumn{2}{|c|}{ PW (18) } \\
\hline & & & & & Yes & No & Yes & No \\
\hline & PTV n & lose & Hot sp & & & & & \\
\hline & MFIF & PW & MFIF & PW & & & & \\
\hline & (8) & $(18)$ & (8) & $(18)$ & & & & \\
\hline Axillary & & & $\begin{array}{l}2 \\
4\end{array}$ & & $2(100 \%)$ & 0 & $4(100 \%)$ & 0 \\
\hline Breast & 3 & 8 & & & $2(65 \%)$ & $1(33 \%)$ & $\begin{array}{l}6(75 \%) \\
2(25 \%)\end{array}$ & \\
\hline Inframammary fold & 1 & 2 & $\begin{array}{l}1 \\
3\end{array}$ & & 0 & $2(100 \%)$ & $\begin{array}{l}2(40 \%) \\
3(60 \%)\end{array}$ & \\
\hline $\begin{array}{l}\text { Sternal / } \\
\text { Parasternal }\end{array}$ & & & $\begin{array}{l}1 \\
1\end{array}$ & & 0 & $1(100 \%)$ & $\begin{array}{l}0 \\
1(100 \%)\end{array}$ & \\
\hline
\end{tabular}

Table 5: The distribution of the high dose regions and its relationship to areas of moist desquamation. Numbers in parentheses are number of patients who developed moist desquamation in MFIF and PW respectively. using PW plans compared to only 8 patients (53.3\%) of the same breast PTV volume treated using multiple MFIF plans $(\mathrm{P}<0.01)$.

\begin{tabular}{|l|l|l|l|l|}
\hline & $\begin{array}{l}\text { Multiples } \\
\text { Fields in Fields } \\
\text { (Study group) } \\
\text { Mean } \pm \text { SD }\end{array}$ & $\begin{array}{l}\text { PW } \\
\text { (Control } \\
\text { group) } \\
\text { Mean } \pm \text { SD }\end{array}$ & $\begin{array}{l}\text { P } \\
\text { Volume }\end{array}$ & 95\% Cl \\
\hline Over 107\% in PTV & $5.2 \pm 1$ & $8.4 \pm 1.2$ & $\mathrm{P}<0.01$ & $2.7-3.5$ \\
\hline $\begin{array}{l}\text { Over 20 Gy in } \\
\text { ipsilateral lung }\end{array}$ & $7.3 \pm 0.35$ & $7.5 \pm 0.67$ & $\mathrm{P}=0.134$ & $-0.27-0.31$ \\
\hline $\begin{array}{l}\text { Over 2 Gy in } \\
\text { contralateral } \\
\text { breast }\end{array}$ & $1.2 \pm 0.2$ & $2.3 \pm 0.5$ & $\mathrm{P}<0.01$ & $0.96-1.2$ \\
\hline Over 40 Gy in heart & $0.97 \pm 0.9$ & $1.1 \pm 0.7$ & $\mathrm{P}=0.096$ & $-0.6-0.45$ \\
\hline
\end{tabular}

Table 4: The percentage volumes of doses over 107\% in PTV and the volumes over the constraint dose of OARs.

We also correlated the distribution of the hot spot locations receiving $>100 \%$ of prescribed dose and maximum target dose within breast PTV to areas of moist desquamation. The breast area is the most common location for maximum target dose while the axillary area, inframammary fold, and sternal/parasternal areas are common location for hot spot foci outside breast PTV in both MFIF and PW treated patients as shown in Table 5. The majority of patients with moist desquamation over the breast or axillary areas had the high dose regions located at these two areas in both MFIF and PW treated patients. On the other hand, the occurrence of moistdesquamation at the inframammary fold or sternal areas are not so correlated with the high dose regions. 
Citation: Zaghloul HA, Rashed YA, Shaukat AA, Rostom YA, Badawy SE (2014) Intensity Modulated Radiotherapy Using Multiple in Fields Compared to Wedged Fields in Breast Irradiation: Clinical Impact on Early Skin Reaction and Organs at Risk Doses. J Nucl Med Radiat Ther 5: 176. doi:10.4172/2155-9619.1000176

Page 6 of 8

Multiple regression analysis revealed that acute skin reaction in both groups was significantly related to radiation therapy technique (PW and MFIF) $(\mathrm{P}=.0002)$, maximum dose with in the PTV $(\mathrm{P}<0.01)$ medial tangential fields monitor unit.

$(\mathrm{P}=0.011)$ and whole breast PTV volume $(\mathrm{P}<0.01)$.

\section{Discussion}

An investigation into a range of published modulation methods of breast IMRT, including inverse-planning and different types of forward planning including plane compensation, beam's eye view dose contouring, minimization of dose variation and equalization of maximum dose algorithms, was conducted. The majority of these methods improved dose uniformity over wedge-only plans especially for breast sizes of $500 \mathrm{~cm}^{3}$ or greater. However, MFIF using beam 's eye view (BEV) isodose-contouring and equalization of the maximum dose achieved the best reduction of high-dose volumes, without clear dosimetric advantage of other methods of intensity modulation including inverse IMRT over it [10].

In the present study, we evaluated the impact of MFIF using beam `s eye view (BEV) isodose-contouring on various treatment dosimetric parameters, incidence of acute skin reaction and organs at risk doses compared to PW. There was a significant reduction in both the hot spot values and PTV volume covered by maximum target dose in the MFIF plans when compared to PW plans $(107.6 \%$ vs $109.4 \%, \mathrm{P}<0.01)$ and $(6.4 \%$ vs $9.4 \%, \mathrm{P}<0.001)$ respectively. Similarly, Guillian et al. concluded that there was a statistically significant improvement in both the absolute volume receiving $>107 \%(\mathrm{Vol}>107)$ and the absolute volume receiving $<95 \%$ ( $\mathrm{Vol}<95$ ) of the prescribed dose in the forward (IMRT) group when compared to the PW group [20]. De la Torre et al. also reported that MFIF technique allowed for better dose uniformity and the elimination of hot spots [21]. The mean values of the homogeneity index $(\mathrm{HI})$ were significantly improved in MFIF compared to PW plans $(0.83 \pm 0.7$ vs $0.94 \pm 0.1)(\mathrm{P}<0.001)$ as the lesser value of $\mathrm{HI}$ in MFIF indicated a significantly greater $3 \mathrm{D}$ dose homogeneity in the PTV. More interestingly, Cem et al. concluded that Dose HI was significantly lower for the FIF $(0.117 \pm 0.021)$ than for the single wedge $(0.131 \pm 0.025, \mathrm{P}=0.02)$ and double wedged plan $(0.128 \pm 0.025, \mathrm{P}=0.04)$, respectively [22]. Concerning TLD measured skin surface dose, MFIF plans reduced contralteral breast surface dose compared to PW (11.4 cGy vs $13.7 \mathrm{cGy}$ respectively). Moreover, MFIF delivered none significantly higher mean doses on the surface of the treated breasts than PW (129.5 cGy vs 123.4 cGy respectively). Our results were consistent with de la Torre et al as they proved that MFIF reduced contralateral breast surface dose compared to PW [21]. More interestingly, the percent volume of the contralateral breast receiving over 2 Gy was significantly reduced $(1.2 \pm 0.2$ vs to $2.3 \pm 0.5)$ in MFIF compared to $\mathrm{PW}$ plans respectively $(\mathrm{P}<0.01)$. A siginifcant reduction in the contralateral breast doses per 2-Gy fraction from 7.3 cGy \pm 2.1 cGy in wedged tangential (WT), to $4.7 \mathrm{cGy} \pm 1.9 \mathrm{cGy}$ in planned forward segements (ST); $\mathrm{p}<0.01$ ) was also reprted by Ludwig et al. in their dosimetric study [23]. With regards to other organs at risk : lung V20, heart V30 in MFIF treated patients were significantly reduced compared to $\mathrm{PW}$ treated patients $10.6 \%$ vs $14.3 \%(\mathrm{P}<0.01)$ and $6 \% \mathrm{vs}$ $7 \% \quad(\mathrm{P}<0.001)$ respectively. Ohashi et al. reported a significant reduction in lung V20 from $8.9 \%$ to $7.4 \%(\mathrm{p}<0.01)$, and heart V30 from $15.9 \mathrm{ml}$ to $7.6 \mathrm{ml}(\mathrm{p}<0.01)$ for PW when compared to MFIF [23].

Our study confirmed a significant reduction in the incidence of grade 2 acute skin reaction as it occurred in $32 \%$ in MFIF vs $61.3 \%$ in
PW $(\mathrm{P}<0.01)$. Similary, Harsolia et al. confirmed a significant reduction in acute grade 2 skin reaction with IMRT compared with wedges ( $41 \%$ vs $85 \%$ respectively, $\mathrm{P}<0.01$ ). More intersringly, in the present study, acute grade 3 skin reaction (moist desquamation) was significantly reduced in MFIF compared to PW (10.7\% vs $24 \%$ $\mathrm{P}<0.01)$, while Harsolia et al. reported only, a trend toward reduced acute grade 3 or greater dermatitis $(6 \%$ vs. $1 \%, \mathrm{P}=0.09)$ in favor of IMRT vs PW [24,25]. A possible explanation of the reduced incidence of grade 3 dermatitis might be related to the better matching between MFIF and PW with no significant difference in breast volumes, beam energies or other parameters between the treatment groups based on binary logistic regression. On the other hand, Harisola et al. did not clarify how they matched patients in both treatment groups (IMRT vs PW) to avoid bias in their results. Moreover, they mentioned that patient in PW were treated using 2-D planning and there was a significant difference in number of patient treated with $6 \mathrm{MV}$ beam in PW compared to IMRT who were mainly treated with mixed 6 and 18 MV beams. Additionally, the tumor bed boost was $10 \mathrm{~Gy} / 5$ fractions in our study compared to $16 \mathrm{~Gy} / 8$ fractions in Harsolia et al study. We also observed that the majority of patients with moist desquamation over the breast or axillary areas had the high dose regions located at these two areas in both MFIF and PW treated patients. Similarly, Sun et al., concluded a relation between the distribution of the hot spot locations and most areas of moist desquamation following whole breast irradiation [26].

Our study proved that clinical application of the MFIF using beam's eye view (BEV) isodose-contouring and equalization of the maximum dose, achieved significant improved performance when compared to the effect of conventional PW technique. It significantly enhanced the dose homogeneity index, it reduced the maximum dose covering the PTV and the PTV volume covered by the maximum dose. Moreover it reduced doses to organs at risks (lung, heart, contralateral breast) and finally, reduced incidence of grade 2 grade 3 acute skin.

There are several sentinel studies scrutinizing on the use of inverse IMRT to improve dose distributions with whole breast irradiation [25,27-30]. However, the increase in rad-on time in IMRT resulted in higher bone marrow exposure to scattered doses. Moreover, the significant cost and requirement of human resources demanded by the implementation of the most advanced IMRT should be considered meticulously $[13,14]$. On the contrary, the MFIF technique did not require a lot of resources for treatment planning and delivery [27]. Furthermore, the MFIF technique does not require a pretreatment QA procedure, which is essential for IMRT [10,27,28].

There were limitations to our study. First, our study was a retrospective analysis with limited number of patients, which potentially limits the generalizability of our results to results of prospective studies. Second, Comparing the MFIF treated patient one to one to a matched control group of patient treated using PW was subjected to the effect of confounding variables. Patients in the control (PW) group were individually matched to the MFIF patients based on age, breast size, TNM staging, chemotherapy regimen and hormonal treatment given.

Fortunately, with individual matching the problem of confounding variables was much reduced, although it was difficult to find an individually matched control. Moreover, the effect of confounding variables was further adjusted by using multivariate analysis. We are planning to extend the follow up duration of the whole study population to two years to further evaluate the impact of significantly 
Citation: Zaghloul HA, Rashed YA, Shaukat AA, Rostom YA, Badawy SE (2014) Intensity Modulated Radiotherapy Using Multiple in Fields Compared to Wedged Fields in Breast Irradiation: Clinical Impact on Early Skin Reaction and Organs at Risk Doses. J Nucl Med Radiat Ther 5: 176. doi:10.4172/2155-9619.1000176

Page 7 of 8

reduced acute skin reaction achieved by MFIF compared to PW on cosmetic outcome.

\section{Conclusion}

The MFIF technique significantly improved PTV dose homogeneity index, while significantly reduced the incidence of grade $2 / 3$ acute skin reaction and doses to OARs when compared to PW tangential fields in whole breast radiotherapy.

\section{Conflict of Interest Statement}

\section{All authors}

Hala Ahmed Zaghloul El Lathy, MSc, MD. Radiation Oncology King Fahad Specialist Hospital Al Dammam, KSA \& Radiation Oncology, Alexanderia University, Egypt.

Y. Rashed, PhD Radiation Physics. Radiation Oncology King Fahad Specialist Hospital Al Dammam, KSA.

A. A Shaukat, FRCR. Radiation Oncology King Fahad Specialist Hospital Al Dammam, KSA.

Y.A.Rostom, MSc, MD. Radiation Oncology, Alexanderia University, Egypt.

Samy El Badawy, FRCR. Radiation Oncology King Fahad Specialist Hospital Al Dammam, KSA.

All authors declare that there was no conflict of interest on conducting this study. They also confirm that no financial support nor grants were given to conduct this study.

\section{Ethics Standard}

The study has been approved by our institutional ethics committee and have therefore been performed in accordance with the ethical standards laid down in the 1964 Declaration of Helsinki and its later amendments. All procedures followed were in accordance with the ethical standards of the responsible committee on human experimentation (institutional and national) and with the Helsinki Declaration of 1975, as revised in 2008.

\section{Informed Consent}

All persons gave their informed consent prior to their inclusion in the study. Details that might disclose the identity of the subjects under study all were omitted. Include the following: Informed consent was obtained from all patients for being included in the study.

\section{References}

1. Aref A, Thornton D, Youssef E, He T, Tekyi-Mensah S, et al. (2000) Dosimetric improvements following 3D planning of tangential breast irradiation. Int J Radiat Oncol Biol Phys 48: 1569-1574.

2. Buchholz TA, Gurgoze E, Bice WS, Prestidge BR (1997) Dosimetric analysis of intact breast irradiation in off-axis planes. Int J Radiat Oncol Biol Phys 39: 261-267.

3. Moody AM, Mayles WP, Bliss JM, A'Hern RP, Owen JR, et al. (1994) The influence of breast size on late radiation effects and association with radiotherapy dose inhomogeneity. Radiother Oncol 33: 106-112.

4. Vicini FA, Sharpe M, Kestin L, Martinez A, Mitchell CK, et al. (2002) Optimizing breast cancer treatment efficacy with intensity-modulated radiotherapy. Int J Radiat Oncol Biol Phys 54: 1336-1344.
5. Mayles WP, Yarnold JR, Webb S (1991) Improved dose homogeneity in the breast using tissue compensators. Radiother Oncol 22: 248-251.

6. Donovan EM, Johnson U, Shentall G, Evans PM, Neal AJ, et al. (2000) Evaluation of compensation in breast radiotherapy: a planning study using multiple static fields. Int J Radiat Oncol Biol Phys 46: 671-679.

7. Lo YC, Yasuda G, Fitzgerald TJ, Urie MM (2000) Intensity modulation for breast treatment using static multi-leaf collimators. Int J Radiat Oncol Biol Phys 46: 187-194.

8. Kestin LL, Sharpe MB, Frazier RC, Vicini FA, Yan D, et al. (2000) Intensity modulation to improve dose uniformity with tangential breast radiotherapy: initial clinical experience. Int J Radiat Oncol Biol Phys 48: 1559-1568.

9. Richmond ND, Turner RN, Dawes PJ, Lambert GD, Lawrence GP (2003) Evaluation of the dosimetric consequences of adding a single asymmetric or MLC shaped field to a tangential breast radiotherapy technique. Radiother Oncol 67: 165-170.

10. Donovan EM, Yarnold JR, Adams EJ, Morgan A, Warrington AP, et al. (2008) An investigation into methods of IMRT planning applied to breast radiotherapy. Br J Radiol 81: 311-322.

11. Freedman GM, Anderson PR, Li J, Eisenberg DF, Hanlon AL, et al. (2006) Intensity modulated radiation therapy (IMRT) decreases acute skin toxicity for women receiving radiation for breast cancer. Am J Clin Oncol 29: 66-70.

12. Pignol JP, Olivotto I, Rakovitch E, Gardner S, Sixel K, et al. (2008) A multicenter randomized trial of breast intensity-modulated radiation therapy to reduce acute radiation dermatitis. J Clin Oncol 26: 2085-2092.

13. Haffty BG, Buchholz TA, McCormick B (2008) Should intensitymodulated radiation therapy be the standard of care in the conservatively managed breast cancer patient? J Clin Oncol 26: 2072-2074.

14. Stillie AL, Kron T, Herschtal A, Hornby C, Cramb J, et al. (2011) Does inverse-planned intensity-modulated radiation therapy have a role in the treatment of patients with left-sided breast cancer? J Med Imaging Radiat Oncol 55: 311-319.

15. Lee JW, Hong S, Choi KS, Kim YL, Park BM, et al. (2008) Performance evaluation of field-in-field technique for tangential breast irradiation. Jpn J Clin Oncol 38: 158-163.

16. ICRU (1993) Prescribing, recording, and reporting photon beam therapy. ICRU Report 50. Bethesda, Maryland: International Commission on Radiation Units and Measurements.

17. ICRU (1999) Prescribing, recording, and reporting photon beam therapy (supplement to ICRU Report 50). ICRU Report 62. Bethesda, Maryland: International Commission of Radiation Units and Measurements.

18. Murthy KK, Sivakumar SS, Davis CA, Ravichandran R, El Ghamrawy K (2008) Optimization of dose distribution with multi-leaf collimator using field-in-field technique for parallel opposing tangential beams of breast cancers. J Med Phys 33: 60-63.

19. Cancer Therapy Evaluation Program. Common terminology criteria for adverseevents: NationalCancerInstitute.

20. Barnett GC, Wilkinson J, Moody AM, Wilson CB, Sharma R, et al. (2009) A randomised controlled trial of forward-planned radiotherapy (IMRT) for early breast cancer: baseline characteristics and dosimetry results. Radiother Oncol 92: 34-41.

21. de la Torre N, Figueroa CT, Martinez K, Riley S, Chapman J (2004) A comparative study of surface dose and dose distribution for intact breast following irradiation with field-in-field technique vs. the use of conventional wedges. Med Dosim 29: 109-114.

22. Onal C, Sonmez A, Arslan G, Oymak E, Kotek A, et al. (2012) Dosimetric comparison of the field-in-field technique and tangential wedged beams for breast irradiation. Jpn J Radiol 30: 218-226.

23. Ludwig V, Schwab F, Guckenberger M, Krieger T, Flentje M (2008) Comparison of wedge versus segmented techniques in whole breast irradiation: effects on dose exposure outside the treatment volume. Strahlenther Onkol 184: 307-312.

24. Ohashi T, Takeda A, Shigematsu N, Fukada J, Sanuki N, et al. (2009) Dose distribution analysis of axillary lymph nodes for three-dimensional 
Citation: Zaghloul HA, Rashed YA, Shaukat AA, Rostom YA, Badawy SE (2014) Intensity Modulated Radiotherapy Using Multiple in Fields Compared to Wedged Fields in Breast Irradiation: Clinical Impact on Early Skin Reaction and Organs at Risk Doses. J Nucl Med Radiat Ther 5: 176. doi:10.4172/2155-9619.1000176

Page 8 of 8

conformal radiotherapy with a field-in-field technique for breast cancer Int J Radiat Oncol Biol Phys 73: 80-87.

25. Harsolia A, Kestin L, Grills I, Wallace M, Jolly S, et al. (2007) Intensitymodulated radiotherapy results in significant decrease in clinical toxicities compared with conventional wedge-based breast radiotherapy. Int J Radiat Oncol Biol Phys 68: 1375-1380.

26. Sun LM, Huang EY, Liang JA, Meng FY, Chang GH, et al. (2013) Evaluation the consistency of location of moist desquamation and skin high dose area for breast cancer patients receiving adjuvant radiotherapy after breast conservative surgery. Radiat Oncol $8: 50$.

27. Donovan E, Bleakley N, Denholm E, Evans P, Gothard L, et al. (2007) Randomised trial of standard 2D radiotherapy (RT) versus intensity modulated radiotherapy (IMRT) in patients prescribed breast radiotherapy. Radiother Oncol 82: 254-264.
28. Donovan EM, Bleackley NJ, Evans PM, Reise SF, Yarnold JR (2002) Dose-position and dose-volume histogram analysis of standard wedged and intensity modulated treatments in breast radiotherapy. Br J Radiol 75: 967-973.

29. Mayo CS, Urie MM, Fitzgerald TJ (2005) Hybrid IMRT plans-concurrently treating conventional and IMRT beams for improved breast irradiation and reduced planning time. Int J Radiat Oncol Biol Phys 61: 922-932.

30. Barnett GC, Wilkinson JS, Moody AM, Wilson CB, Twyman N, et al. (2012) Randomized controlled trial of forward-planned intensity modulated radiotherapy for early breast cancer: interim results at 2 years. Int J Radiat Oncol Biol Phys 82: 715-723. 